\title{
DYNAMIC RESOURCE PROVISIONING FOR SELF-ADAPTIVE HETEROGENEOUS WORKLOADS IN SMP HOSTING PLATFORMS
}

\author{
Ramon Nou, Ferran Julià, Jordi Guitart and Jordi Torres \\ Barcelona Supercomputing Center(BSC), Technical University of Catalonia (UPC), Barcelona, Spain \\ rnou@ac.upc.edu,ferran.julia@bsc.es, jguitart@ac.upc.edu,torres@ac.upc.edu
}

Keywords: Autonomic Computing, resource provisioning, heterogeneous workloads.

\begin{abstract}
We introduce a novel approach that allows heterogeneous applications run together on a shared hosting platform, dynamically sharing the platform's resources. The proposed approach has been validated by a proofof-concept prototype which uses a global processor manager to distribute the platform's processors among two (or more) heterogeneous applications, i.e. a Tomcat application server and a Globus grid middleware. Our evaluation demonstrates the benefit of including bidirectional communication between applications and the OS for efficiently managing the resources and preventing the degradation of an applications performance, especially when the hosting platform is fully overloaded. For the sake of simplicity, we have modified the applications so that they communicate with the resource manager, although other techniques can be applied to avoid these modifications. Running different applications in a shared platform and being able to assign priorities between them provides important benefits.
\end{abstract}

\section{INTRODUCTION}

The consolidation of distributed and grid computing has been accompanied with the appearance of new computing models oriented to these environments. One of them is the utility computing model, in which applications run on hosting platforms that rent their resources to them. Application owners pay for platform resources, and in return, the application is provided with guarantees of resource availability and quality of service (QoS), which can be expressed in the form of a service level agreement (SLA). The hosting platform is responsible for providing sufficient resources to each application to meet its workload, or at least to satisfy the agreed QoS. These hosting platforms must be able to provide resources to a heterogeneous set of applications, which range from web applications (e.g. an application server attending a transactional workload) to traditional scientific computations in the grid community. The traditional approach used by hosting platforms for provision of resources to heterogeneous applications is to consider a separate set of the cluster nodes for each application (dedicated model) (Appleby et al., 2001). In this model, resource allocation is performed with the granularity of a full cluster node and the provisioning technique must determine how many nodes to allocate to each application. However, economic reasons of space, power, cooling and cost can encourage the use of the shared model (Chandra et al., 2003a), in which node resources can be shared among multiple applications and the provisioning technique needs to determine how to partition resources on each node among competing applications. We introduce a novel approach to allow heterogeneous applications to run together in a shared hosting platform, dynamically sharing the platform's resources (we focus on CPUs in this proof-of-concept prototype) while maintaining good performance. This paper extends the work performed in (Guitart et al., 2006). The previous paper proposed a global strategy for preventing the overloading of web applications and efficiently utilizing a platform's resources in a shared hosting platform running homogeneous web applications. The proposed strategy exploits the benefits of dynamically reallocating resources among hosted applications based on the variations in their workloads. These benefits have been described in recent studies (Appleby et al., 
2001; Chandra et al., 2003a; Chandra et al., 2003b). The goal is to meet the applications requirements on demand and adapt to their changing resource needs. This requires an accurate collaboration between dynamic resource provisioning and admission control mechanisms. The paper is structured as follows: Section 2 shows our prototype and how it works. Section 3 describes the experimental environment used in our evaluation. Section 4 evaluates the results we obtained. Section 5 explores some work done in the area of dynamic resource provisioning for variable workloads. Finally, section 6 presents our conclusions and future work.

\section{RESOURCE PROVISIONING STRATEGY}

In this section, we present a summary of the basic guidelines of our resource provisioning strategy. Our proposal is based on a global processor manager, called eDragon CPU Manager (ECM), responsible for periodically (configurable) distributing the available processors among the different applications running in a hosting platform. Further details can be found in (Guitart et al., 2006). We manage only processors because are the more limited resource in the scenario we are studying. The ECM cooperates with the applications to efficiently manage the processors and prevent applications getting overloaded using bidirectional communication. On one side, the applications periodically request from the ECM the number of processors needed to handle their incoming load while avoiding degradation in the QoS. We define the number of processors requested by an application $i$ as $R_{i}$. On the other side, the ECM can be requested at any time by the applications to inform them about their processor assignments. We define the number of processors allocated to application $i$ as $A_{i}$. With this information, the applications can adapt their behavior to the allocated processors, avoiding in this way the degradation of their QoS. Figure 1 shows a diagram describing our resource provisioning strategy.

\section{1 eDragon CPU Manager}

The eDragon CPU Manager (ECM) is responsible for the distribution of processors among applications in the hosting platform. The ECM is implemented as a user-level process that wakes up periodically at a fixed time quantum, defined as $k_{E C M}$, examines the current requests of the applications and distributes processors according to a scheduling policy. With this configuration, direct modification of the native kernel is not

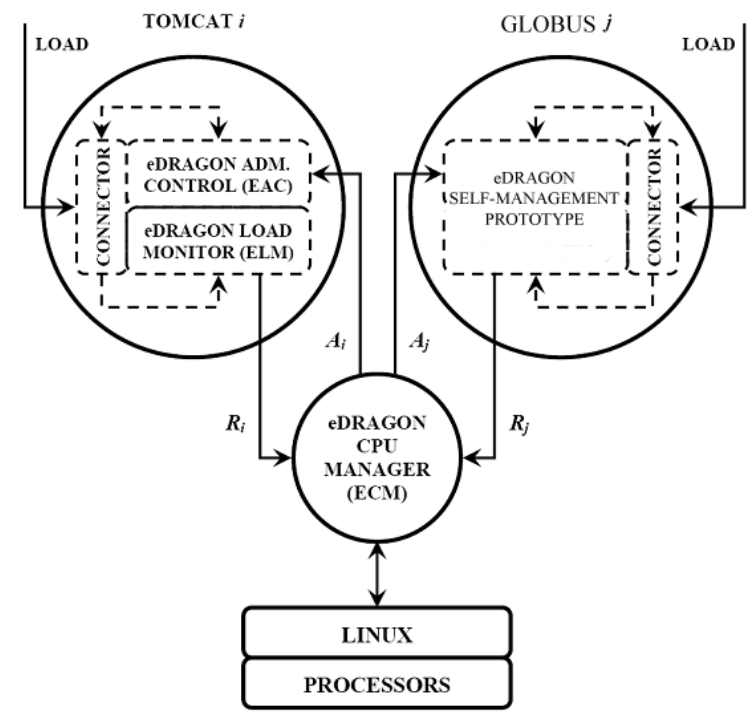

Figure 1: Prototype structure with ECM and two applications

required to show the usefulness of the proposed environment.

Traditionally, resource allocation policies have considered conventional performance metrics such as response time, throughput and availability. However, the metrics that are of utmost importance to the management of an e-commerce site are revenue and profits and should be incorporated when designing policies (Cherkasova and Phaal, 2002). For this reason, the ECM can implement policies considering conventional performance metrics as well as incorporating ebusiness indicators, also policies like the ones in Section 5 can also be incorporated to the ECM. Our sample policy includes priority classes. The priority class $P_{i}$ indicates a customer domain's priority in relation to other customer domains. It is expected that high priority customers will receive preferential service respect low priority customers. In our policy, at every sampling interval $k_{E C M}$, each application $i$ receives a number of processors $\left(A_{i}\left(k_{E C M}\right)\right)$ that is proportional to its request $\left(R_{i}\left(k_{E C M}\right)\right)$ pondered depending on the application's priority class $\left(P_{i}\right)$ and the number of processors in the hosting platform (NCpus), and inversely proportional to the total workload of the system $\left(\sum P_{j} * R_{j}\left(k_{E C M}\right)\right)$, expressed as the sum of requests of all applications in the system. The scheduling policy should also allow us to achieve the highest resource utilization in the hosting platform. Our proposal to accomplish this with the ECM is based on sharing processors among the applications under certain conditions (minimizing the impact on performance isolation). 
The ECM not only decides how many processors to assign to each application, but also which processors to assign to each application. In order to accomplish this, the ECM configures the CPU affinity mask of each application (using the Linux sched setaffinity function) so that the processors allocations to the different applications do not overlap (except if one processor is shared), in this way minimizing the performance interference among applications.

\section{EXPERIMENTAL ENVIRONMENT}

We have Tomcat v5.0.19 (Amza et al., 2002) and a Globus GT 4.0.1 (Sotomayor and Childers, 2005) in the same node. Tomcat is an open-source servlet container developed under the Apache license. Its primary goal is to serve as a reference implementation of the Sun Servlet and JSP specifications, and to be a quality production servlet container too.

The client workload for the experiments was generated using a workload generator and web performance measurement tool called Httperf (Crovella et al., 1999) using RUBiS (Rice University Bidding System) (Coarfa et al., 2002) benchmark servlets as an application. The Tomcat instance has a variable input load throughout the run time, which is shown in the top subfigure of Figure 2 which displays the number of new clients per second that hit the server as a function of the time. Input load distribution has been chosen in order to represent the different processor requirement combinations when running with the Grid workload in the hosting platform.

The Globus server is a standard de facto of Grid middleware. We didn't make any modifications of its parameters (number of ServiceThreads or number of Runqueues) on the standard tests (with ECM they can be dynamically modified). The Globus workload generator is sending jobs that overload or stress the management code for a job. We assume that the job will be executed on another node or cluster. From the Globus workload generator we generate and submit jobs with an increasing throughput and try to execute them on the nodes of the cluster (simulated, so CPU requeriments are only the ones to prepare the job). We then measure the output throughput. This gives a wide range of situations that are summarized in Figure 2. In our case, we selected different submission levels for Globus and several different levels of arrival rates for Tomcat to give a wide view of configurations and situations to show the benefits of our approach. The hosting platform is a 4-way Intel XEON 1.4 GHz with 2 GB RAM.
For the purpose of this paper, we present a managed middleware prototype that allows the execution of heterogeneous applications in the same hosting platform. We show how the communication between applications and OS layer can provide great improvements in performance terms. For this proof-ofconcept we are considering web and grid applications. For simplicity in the prototype, we are using Tomcat for the web workload and the Globus platform for the grid workload; all of them are well known platforms and widely used. In this prototype we are modifying the applications in order to communicate with ECM, but we could use other mechanism to avoid these modification (i.e. use a proxy). Further details of the architecture and modifications done on Tomcat and Globus, can be seen on (Guitart et al., 2006) for Tomcat and (Nou et al., 2007a; Nou et al., 2007b; IBM-Corporation, 2004) for Globus Toolkit.

\section{EVALUATION}

Our evaluation will show the benefits of our proposal for managing the resources efficiently and preventing server overload on a 4-way multiprocessor Linux hosting platform. The CPU requeriments to prepare a globus job are ten times the requeriments to process a Tomcat request (with SSL handshake) or 100 times a Tomcat request without SSL handshake.

\subsection{Standard Tomcat and Globus}

In Figure 2, we can see in grid style how the system evolves (bottom two plots) when we are submitting different workloads (top plot) to Tomcat and Globus. We have divided the test in order to check different CPU requirement scenarios in the two middleware. Globus is submitting the jobs with the increasing workload explained in Section 3, while Tomcat is generating several load levels from overloaded to non-overloaded arrival rates. As we can see in the top plot, we can divide the test into two parts; one workload with low load and another workload where the system is under a heavy load. If we take a closer view of Tomcat we can see that when the server is overloaded the reply/rate falls (i.e. around $4000 \mathrm{sec}$ onds in grid style). We can find another zone in the Tomcat plot of similar behaviour: in the 1500 seconds point the throughput obtained from Tomcat is very high, however when we look at the 3000 seconds zone we can see that the throughput obtained is lower with an higher input workload. Switching to the Globus plot there are a lot of zones where no jobs are finished (2800-3600 or 4000-5000). Globus is getting a very 

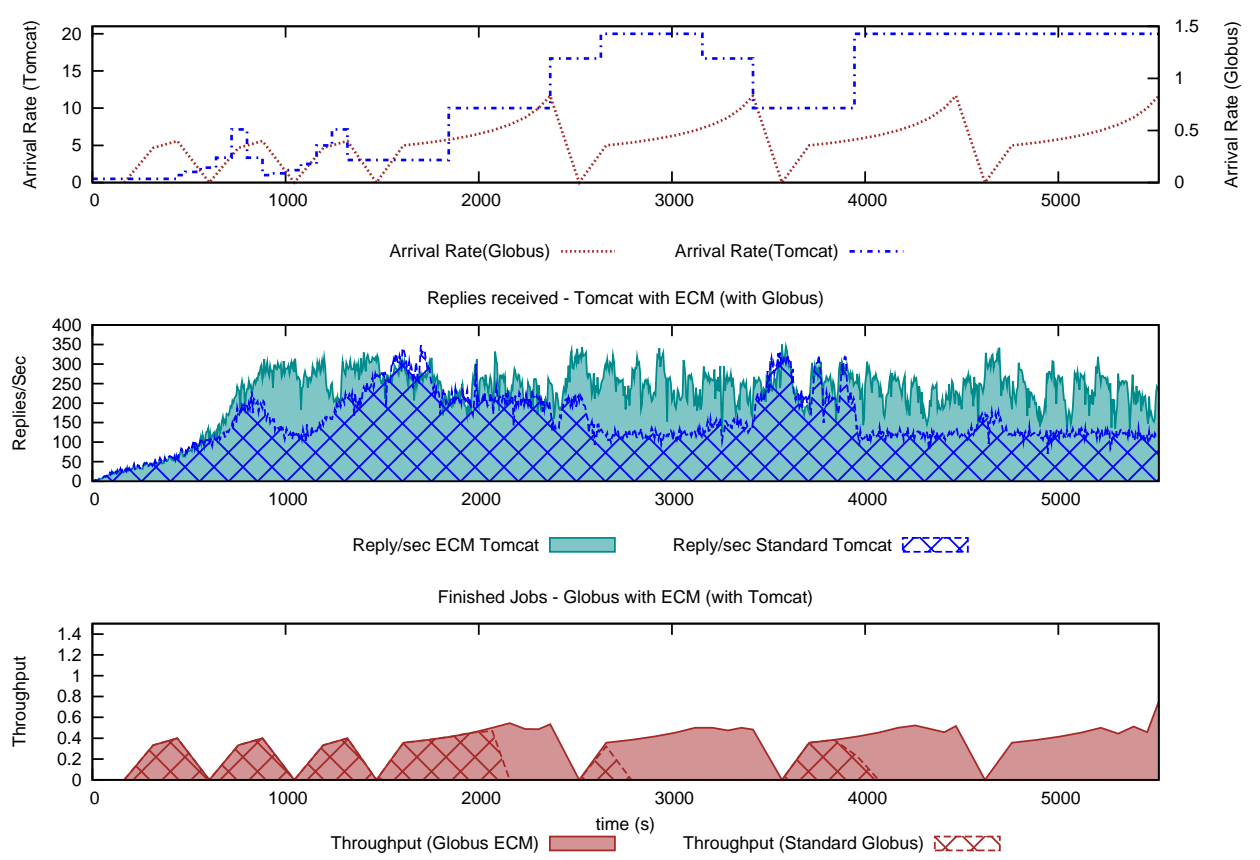

Figure 2: From top to bottom; workload of Tomcat and Globus, replies/sec of Standard Tomcat compared with the replies/sec of Tomcat using ECM, throughput in Standard Globus compared with throughput using ECM with Globus. The two applications are running at once using ECM facilities

high workload, but as we will show in the next subsection we can increase its performance using ECM. The server needs to share the resources between the two applications, but such kinds of applications don't know in which environment and with what kinds of resources they are being executed. They are competing for a set of limited resources and getting worse performance than if they were divided onto two machines with the resources divided in half. Giving and getting information about the resources that the application consumes and that the system have available for it should be necessary in order to avoid this situations.

\subsection{Adaptative Middleware with ECM}

If we repeat the last test with ECM (without priorities), we obtain the solid style results in Figure 2. We can see how the system overall is working better. And the most important thing is that we didn't get the low levels of replies/sec on Tomcat and the low number of finished jobs on Globus that we obtained in the previous subsection.

In some zones we can see how Tomcat is working better than before, as long as ECM receives a request of processing power from Tomcat, ECM tells Tomcat how many CPUs has assigned. Tomcat is able to overcome these situations and start its admission control to stabilize itself at the desired level. It is the case of the zones near 1000 seconds and the ranges from 2200-3500 and 4000-5500 where the system without ECM is obtaining lower performance than with ECM. In this zones as long as Tomcat (and Globus) knows how many resources they have available can adapt his behaviour to the new scenario. We can notice this looking at the globus side, where we are getting more throughput than before also. Getting into these situations, on 1000 seconds zone Globus is working at the same level than before but Tomcat as it knows how many resources it has available can adapt his load to the new scenario. The reverse situations happens on 2500 seconds zone, Globus is improving its performance. When the two applications are overloaded a communication with the OS to know the available resources can provide an improvement over the two applications as we can see after 4000 seconds zone. $n$ the other hand we can modify the fairness of the resource assignment using priorities dependending, i.e. the revenue of the application, using ECM. When the applications are in the same priority category inside ECM, they are sharing resources without any preference between applications. In an entry node with a secure connection scenario, like the one we are testing, it's crucial to provide more fairness to the several 
middleware that share the resources on the node to get better results as a whole.

\section{RELATED WORK}

Recent studies (Andrzejak et al., 2002; Chandra et al., 2003b; Chandra and Shenoy, 2003) have reported the considerable benefit of dynamically adjusting resource allocations to handle variable workloads. This premise has motivated the proposal of several techniques to dynamically provision resources to applications in on-demand hosting platforms. Depending on the mechanism used to decide the resource allocations, these proposals can be classified into: control theoretic approaches with a feedback element (Abdelzaher et al., 2002), open-loop approaches based on queuing models to achieve resource guarantees (Chandra et al., 2003a; Doyle et al., 2003; Liu et al., 2001) and observation-based approaches that use runtime measurements to compute the relationship between resources and a QoS goal (Pradhan et al., 2002). Control theory solutions require training the system at different operating points to determine the control parameters for a given workload. Queuing models are useful for steady state analysis but do not handle transients accurately. Observationbased approaches are most suited for handling varying workloads and non-linear behaviors. Resource management in a single machine has been covered in (Banga et al., 1999), where authors proposed to use resource containers as an operating system abstraction to embody a resource. In (Liu et al., 2005) authors proposes the design of online feedback control algorithms to dynamically adjust entitlement values for a resource container on a server shared by multiple applications. The problem of provisioning resources in cluster architectures has been addressed in (Appleby et al., 2001; Ranjan et al., 2002) by allocating entire machines (dedicated model) and in (Chandra et al., 2003a; Pradhan et al., 2002; Uragonkar and Shenoy, 2004) by sharing node resources among multiple applications (shared model). Cataclysm (Sotomayor and Childers, 2005) performs overload control by bringing together admission control, adaptive service degradation and dynamic provisioning of platform resources, demonstrating that the most effective way to handle overloading must consider a combination of techniques. In this aspect, that work is similar to our proposal. There are also approaches (Menascé, 2005) that use virtualized environments and analytical methods to adjust the resources allocated to the virtualized systems. R-Opus (Cherkasova and Rolia, 2006) works on a different layer and scale of time.
In our approach we focus on a single server machine which shares different applications and has a low time scale. Also, giving more processing power to an application, such as Tomcat (for example), will not directly produce better performance. The application needs to know how many resources it has available.

\section{CONCLUSIONS}

In this paper we have presented a proof-of-concept prototype for demonstrating that bidirectional communication between applications and OS can provide that heterogeneous applications (running in overloaded conditions) can run together in a shared hosting platform and at the same time maintain their performance. Using a shared hosting platform reduces important costs like space and power.

Our approach is based on implementing a global resource manager, responsible for periodically distributing the available processors between the applications following a determined policy. The resource manager can be configured to implement different policies, and consider traditional indicators (i.e. response time) as well as e-business indicators (i.e. customer's priority). In our proposal, the resource manager and the applications cooperate to manage the resources, in a manner totally transparent to the user, using bi-directional communication. On one side, the applications request from the resource manager the number of processors needed to handle their incoming load without QoS degradation. On the other side, the resource manager can be requested at any time by the applications to inform them about their processor assignments. With this information, applications can adapt their behavior to the allocated processors.

Our evaluation demonstrates the benefit of our approach for managing resources efficiently and for preventing degradation of an applications performance on shared hosting platforms. Although our implementation targets Tomcat and Globus, the proposed strategy can be applied with any other platform or application. Further improvements can be made on this proof-of-concept work: more fine grained assignments of CPU or a fairer Globus self-management objective. Our future work considers the use of virtualization technologies.

\section{ACKNOWLEDGMENT}

This work is supported by the Ministry of Science and Technology of Spain and the European Union 
under contract TIN2004-07739-C02-01 and Commission of the European Communities under IST contract 034286 (SORMA). Thanks to Mario Macias for his help.

\section{REFERENCES}

Abdelzaher, T., Shin, K., and Bhatti, N. (2002). Performance guarantees for web server endsystems: A control-theoretical approach. IEEE TPDS, 13(1):80-96.

Amza, C., Cecchet, E., Chanda, A., Cox, A., Elnikety, S., Gil, R., Marguerite, J., Rajamani, K., and Zwaenepoel, W. (2002). Specification and implementation of dynamic web site benchmarks. WWC-5, Austin, Texas, USA.

Andrzejak, A., Arlitt, M., and Rolia., J. (2002). Bounding the resource savings of utility computing models. HPL-2002-339, HP Labs.

Appleby, K., Fakhouri, S., Fong, L., Goldszmidt, G., Krishnakumar, S., Pazel, D., Pershing, J., and Rochwerger, B. (2001). Oceano :SLA-based management of a computing utility. IM 2001, Seattle, Washington, USA, pages 855-868.

Banga, G., Druschel, P., and Mogul, J. C. (1999). Resource containers: A new facility for resource management in server systems. OSDI'99, New Orleans, Louisiana, USA., pages 45-58.

Chandra, A., Gong, W., and Shenoy, P. (2003a). Dynamic resource allocation for shared data centers using online measurements. IWQoS 2003,Berkeley, California, USA., pages 381-400.

Chandra, A., Goyal, P., and Shenoy, P. (2003b). Quantifying the benefits of resource multiplexing in on-demand data centers. Self-Manage 2003, San Diego, California, USA.

Chandra, A. and Shenoy, P. (2003). Effectiveness of dynamic resource allocation for handling internet flash crowds. TR03-37, Department of Computer Science, University of Massachusetts, USA.

Cherkasova, L. and Phaal, P. (2002). Session-based admission control: A mechanism for peak load management of commercial web sites. IEEE Transactions on Computers, 51 (6):669-685.

Cherkasova, L. and Rolia, J. (2006). R-Opus: A composite framework for application performability and qos in shared resource pools. In $D S N^{\prime} 06$, pages 526-535, Washington, DC, USA.
Coarfa, C., Druschel, P., and Wallach, D. (2002). Performance analysis of TLS web servers. NDSS'02,San Diego, California, USA.

Crovella, M., Frangioso, R., and Harchol-Balter, M. (1999). Connection scheduling in web servers. USITS'99, Boulder, Colorado, USA.

Doyle, R., Chase, J., Asad, O., Jin, W., and Vahdat, A. (2003). Model-based resource provisioning in a web service utility. USITS'03, Seattle, Washington, USA.

Guitart, J., Carrera, D., Beltran, V., Torres, J., and Ayguadè, E. (2006). Preventing secure web applications overload through dynamic resource provisioning and admission control. UPC-DAC$R R-2006-37$.

IBM-Corporation (2004). An architectural blueprint for autonomic computing. http://www.ibm.com/autonomic.

Liu, X., Zhu, X., Singhal, S., and Arlitt, M. (2005). Adaptive entitlement control to resource containers on shared servers. IM 2005, Nice, France.

Liu, Z., Squillante, M., and Wolf, J. (2001). On maximizing service-level-agreement profits. $E C$ 2001, Tampa, Florida, USA., pages 213-223.

Menascé, D. A. (2005). Virtualization: Concepts, applications, and performance modeling. Int. $C M G$ Conference, Orlando, Florida, USA, pages 407414.

Nou, R., Julià, F., and Torres, J. (2007a). The need for self-managed access nodes in grid environments. EASe 2007, Tucson, Arizona, USA.

Nou, R., Julià, F., and Torres, J. (2007b). Should the grid middleware look to self-managing capabilities? ISADS 2007, Sedona, Arizona, USA.

Pradhan, P., Tewari, R., Sahu, S., Chandra, A., and Shenoy, P. (2002). An observation-based approach towards self-managing web servers. IWQoS 2002,Miami Beach, Florida, USA., pages 13-22.

Ranjan, S., Rolia, J., Fu, H., and Knightly, E. (2002). Qos-driven server migration for internet data centers. IWQoS 2002, Miami Beach, Florida, USA., pages 3-12.

Sotomayor, B. and Childers, L. (2005). Globus Toolkit 4 : Programming Java Services. Morgan Kaufmann.

Uragonkar, B. and Shenoy, P. (2004). Cataclysm: Handling extreme overloads in internet services. TR03-40, Department of Computer Science, University of Massachusetts, USA. 